PADs, through a hapten/carrier model. Here, we test this model in mice.

Methods HLA-DRB1*04:01 transgenic mice were immunised subcutaneously with PADs or phosphate buffered saline (PBS) in Freund's complete adjuvant (CFA). Three booster injections of PAD or PBS in Freund' incomplete adjuvant (IFA) were given subcutaneously 15,35 and 55 days later. Mice were:

1. tested for anti-PAD antibodies by ELISA.

2. tested for $\mathrm{T}$ cell responses to PADs, native or citrullinated fibrinogen 65 days after PAD immunisation.

3. tested for anti-citrullinated fibrinogen antibodies by ELISA using fibrinogen peptides under citrullinated and native form.

Results HLA-DRB1*04:01 transgenic mice immunised with PADs developed antibodies and T cells to PADs and IgG antibodies to citrullinated peptides from fibrinogen, in the absence of $\mathrm{T}$ cell response to native or citrullinated fibrinogen.

Conclusions $\mathrm{T}$ cell immunisation to $\mathrm{PAD}$ proteins triggers ACPAs through a hapten carrier mechanism in which the carrier is PAD which performs citrullination and the hapten any protein being citrullinated by PAD.

Disclosure of interest None declared

\section{P013 PORPHYROMONAS GINGIVALIS INFECTION LINKED TO RA ONSET AND ANTI TNF ALPHA TREATMENT NON- RESPONSE}

${ }^{1} \mathrm{M}$ Jenning, ${ }^{1} \mathrm{~B}$ Marklein, ${ }^{2} Y$ Ytterberg, ${ }^{2} \mathrm{~A}$ Catarina, ${ }^{3} \mathrm{D}$ Schaardenburg, ${ }^{1} \mathrm{G}$ Bürmester, ${ }^{1} \mathrm{~K}$ Skriner*. ${ }^{1}$ Department of Rheumatology and Clinical Immunology, Humboldt University and Free University, Berlin, Germany; ${ }^{2}$ Rheumatology Unit, Department of Medicine, Karolinska University Hospital, Stockholm, Sweden; ${ }^{3}$ Amsterdam Rheumatology and Immunology Centre, Reade and Academic Medical Centre, Amsterdam, Netherlands

\subsection{6/annrheumdis-2018-EWRR2018.38}

Introduction Differences in enzymatic activity and pathogenic impact of Porphyromonas gingivalis (P.g.) peptidylarginine deiminase (PPAD) for development of RA have been published, confounded by different PPAD variations and methods used.

Objectives Enzymatic active PPAD isolated from an RA patient (RA-PPAD) was first time linked to citrullination of RA autoantigens, diagnosis, therapy response and RA-onset.

Methods Recombinant RA-PPAD cloned, verified by DNA sequencing and expressed in Escherichia coli and purified. RAPPAD and its enzymatic activity was analysed using 2D-Elektrophoresis, mass spectrometry (MS), immunoblot and ELISA.

Results RA-PPAD autocitrullinates amino acid position 63 $\left(a_{63}\right)$ and exhibits so far two new amino acid mutations $a_{73}$ $\mathrm{F}$ to $\mathrm{L}$ and $\mathrm{aa}_{447} \mathrm{E}$ to $\mathrm{V}$. Anti-citrullinated RA-PPAD antibodies were detected in $38 \%(n=36)$ of patients with RA, but were absent in Systemic lupus erythematosus $(n=30)$, Osteoarthritis $(n=36)$ and control sera $(n=23)$. Twenty percent of RA patients $(n=30)$ showed an increase in antibody-titre against citrullinated RA-PPAD after RA onset. High antibody titre against the cit-PPAD-peptide of 15aa (CPP) derived from the autocitrullination site $\left(\mathrm{R}_{63}\right)$ correlates with $\mathrm{TNF} \alpha$-inhibitor (TBA) non-response $(n=61)$. Anti-CPP levels correlate with DAS28,rheumatoid factor, $\alpha$-CCP 2 levels and increase with age. RA-PPAD is able to citrullinate internal arginines in fibrinogen, vimentin, hnRNP-A2/B1 and histone H1. This internal citrullination-sites are recognised by RA sera and able to bind HLA401.

Conclusions Failure of P.g. clearance in RA patients may lead to excessive exposure of citrullinated self-antigens and bacterial antigens inducing immune-mimicry. P.g. infection can be linked to RA and its correlation to TBA non-response leads to the suggestion to clear P.g infection before $\alpha$-TNF treatment.

Disclosure of interest None declared

\section{P014 FAM167A/BLK IS A SUSCEPTIBILITY LOCUS IN AUTOIMMUNE DISEASES: CHARACTERISATION OF THE FAM167 GENE FAMILY}

L Mentlein*, GE Thorlacius, L Meneghel, Jl Ramirez Sepulveda, S Brauner, A Espinosa, M Wahren-Herlenius. Unit of Rheumatology, Department of Medicine Solna, Karolinska Institute, Solna, Sweden

\subsection{6/annrheumdis-2018-EWRR2018.39}

Introduction Genome-wide association studies of multiple autoimmune diseases, including Sjögren's syndrome, systemic lupus erythematosus and rheumatoid arthritis have identified associations in the FAM167A/BLK locus. eQTL (expression quantitative loci) analyses in peripheral blood cells show a significantly increased expression of FAM167A for the disease associated genotypes. While $B L K$ acts downstream of the $\mathrm{B}$ cell receptor, the function of FAM167A is unknown. Additionally, the role of the only homologous protein, its gene family member FAM167B, remains unexplored.

Objectives To elucidate the potential role of these proteins in susceptibility, or pathogenesis of autoimmune disease, we aim to characterise the FAM167 gene family by both bioinformatic and experimental approaches.

Methods Public databases, including NCBI, GEO, Uniprot, CCLE, PhylomeDB were searched for information on the FAM167 gene family and its two members. The FAM167 protein sequences were analysed with structure prediction tools including YASPIN and D2P2 server. The mRNA levels of the genes of interest were determined by $\mathrm{qPCR}$ analysis on organs from 12 week old C57BL/6 mice and cells of selected human cancer cell lines.

Results After protein sequence analysis we found that the FAM167 gene family shows no homology to any other annotated genes but is highly conserved in vertebrates. The FAM167A and FAM167B proteins don't comprise any previously known protein domains and are predicted to contain both helical and disordered protein structures. Based on the analysis of their protein structure prediction and their conservation we propose that the FAM167 proteins contain two distinct modules linked by a variable linker accounting for the different size of 24 and $18 \mathrm{kDa}$ for FAM167A and B, respectively. Database research revealed that FAM167A is expressed in many cancer cell lines whereas $F A M 167 B$ shows a specifically high expression only within melanoma lines according to CCLE. FAM167A expression was confirmed for the myeloma cell line LP1 and the monocytic cell line THP1 experimentally. High FAM167B expression was found in the melanoma cell line SKMEL28. Expression profiling of mouse organs collected from C57BL/6 mice revealed high expression of Fam $167 a$ in lung, spleen, skeletal muscle and brain. Fam167b had its peak expression in adrenal glands, kidney and liver, with low or no expression observed in other analysed organs.

Conclusions The FAM167A/B genes show distinct expression profiles both at the organ and the cellular level. Further in vitro and in vivo studies will be implemented to unravel the function of this gene family.

Disclosure of interest None declared 